\title{
Airfoil Design via Cubic Splines - Ferguson's Curves Revisited
}

\author{
András Sóbester*, Andy J. Keane ${ }^{\dagger}$ \\ University of Southampton, Southampton, Hampshire, SO17 1BJ, UK
}

\begin{abstract}
Few aerospace design topics have had so many pages devoted to them as airfoil parameterization. Claims of novelty must therefore be made with caution in this area, so we declare our starting point simply as a fresh perspective on legacy techniques, prompted by related developments in design technology. We revisit the Ferguson spline formulation, known since the 1960s, and we propose it as a means of airfoil parameterization, it being ideally suited to implementation in commercial Computer Aided Design (CAD) engines. The development providing the impetus: off-the-shelf CAD tools are taking a widening role in the design process even at its lowest, conceptual levels. We argue that, since similarly constructed splines lie at the heart of modern CAD modeling, the most natural way to describe, say, a wing geometry is via Ferguson-style cubic splines. Further, we show that in the interest of parameterization parsimony, adequate airfoil shape control can be achieved without knots (other than those on the leading and the trailing edge), at the very least at the conceptual level of any design process.
\end{abstract}

\section{Introduction}

$\mathbf{T}$ HE shape of an elastic beam subjected to bending loads is described by a cubic polynomial. In fact, the term spline, now used to describe the family of curves such polynomials belong to, originally described a long, thin strip of wood used by draftsmen to draw a smooth line between specified points (the points that the spline was 'interpolating' were represented by lead weights called ducks). Since the response of a beam to a set of boundary conditions (loads and supports) is fairly easy to picture, this intuitive mathematical behavior contributes to making the cubic spline a very versatile and popular formulation. We encounter them, for example, in statistics (as interpolants and regressors ${ }^{1}$ ), computer graphics (as generators of inbetweens, that is, frames used to fill gaps between given key frames of animations ${ }^{2}$ ) and, most pertinently to the present discussion, in engineering design.

The specific spline formulation that forms our starting point is that of The Boeing Company's James Ferguson, whose seminal 1964 paper $^{3}$ lies at the foundation of the curve (and surface) definition algorithms built into modern Computer Aided Design (CAD) tools. Although Bèzier and others have subsequently published slightly different and now better known forms of Ferguson's cubics, their basic logic remains the same and we shall describe this in Section II. For now, we merely declare the angle from which we approach the problem of airfoil description: we seek to conduct the entire design

\footnotetext{
${ }^{*}$ Lecturer, Computational Engineering and Design Group, AIAA member.

${ }^{\dagger}$ Professor of Computational Engineering, Chair of Computational Engineering and Design Group.
}

1

Submitted to the American Institute of Aeronautics and Astronautics 
process in a commercial CAD world and it therefore makes sense to use the formulations that such software packages allow most naturally.

Additionally, we target the coarse, conceptual stage of the design process, where the search space is large and compactness and parsimony are therefore paramount.

Over the last few decades an impressive array of airfoil description approaches have been developed, including the well-known NACA definitions, the shape function approach of Hicks and Henne, ${ }^{4}$ the orthogonal basis functions of Robinson and Keane, ${ }^{5}$ the $6^{\text {th }}$ order PARSEC polynomials, ${ }^{6}$ various schemes based on knots and/or control points, etc. Kulfan and Bussoletti ${ }^{7}$ (who also propose a generic parameterization method of their own) list eight desirable features an airfoil geometry representation technique should possess. They argue that it should be 1) smooth, well behaved and leading to realistic shapes, 2) mathematically efficient, 3) parsimonious (in terms of the number of design variables), 4) allow the specification of key features (e.g., leading edge radius), 5) allow easy control for editing, 6) it should have an intuitive geometric interpretation, 7) it should be systematic and consistent and 8) robust (they also note that none of the existing approaches ticks all these boxes).

Here we suggest the addition of a ninth criterion, applicable to CAD-based design processes: the representation scheme must be compatible with that of the CAD package. While awkward and often complicated work-arounds enable commercial CAD users to create curves corresponding to most parameterization schemes, the surfaces lofted over them by the CAD engine will inevitably be based on (some flavor of) Ferguson's formulation (b-spline surfaces, NURBS, etc.). The resulting discrepancy will yield ill-defined surfaces, often featuring curvature fluctuations, small gaps and other flaws, whose effect will become painfully obvious at the numerical analysis stage (after all, one of the main functions of the CAD engine is to supply geometric information to analysis codes), if not sooner.

We propose a parameterization method that meets this criterion by definition, while making few compromises on the other eight. After a brief review of the mathematical formulation of Ferguson's splines, we introduce the airfoil description scheme itself (Section III) and examine the extent to which a range of well-known airfoils can be 'cloned' using it. Section IV then considers the most important features of a design space constructed around this parameterization scheme. In Section V we use the method in anger on an airfoil design trade study. Finally, in Section VI we return to the nine criteria mentioned above and we assess our approach against them.

\section{Ferguson's Parametric Splines}

We seek a parametric curve $\mathbf{r}(u)$ with $u \in[0,1]$, connecting two points $\mathbf{r}(0)=\mathbf{A}$ and $\mathbf{r}(0)=\mathbf{B}$. We impose two tangents on the curve: $d \mathbf{r} /\left.d u\right|_{u=0}=\mathbf{T}_{A}$ and $d \mathbf{r} /\left.d u\right|_{u=1}=\mathbf{T}_{B}$, as shown in Figure 1 . We define the curve as the polynomial

$$
\mathbf{r}(u)=\sum_{i=0}^{3} \mathbf{a}_{i} u^{i}, u \in[0,1]
$$




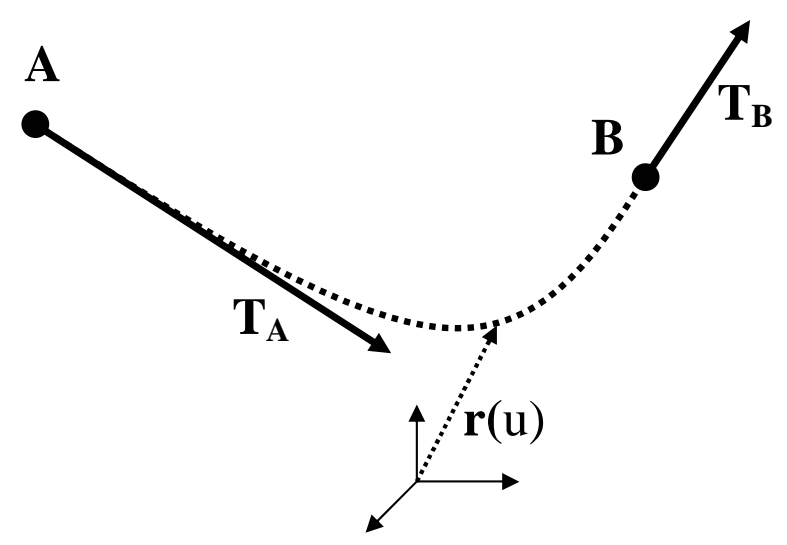

Fig. 1 Ferguson spline and its boundary conditions.

We find the four vectors required to define the curve by setting the endpoint conditions:

$$
\begin{aligned}
& \mathbf{A}=\mathbf{a}_{0} \\
& \mathbf{B}=\mathbf{a}_{0}+\mathbf{a}_{1}+\mathbf{a}_{2}+\mathbf{a}_{3} \\
& \mathbf{T}_{A}=\mathbf{a}_{1} \\
& \mathbf{T}_{B}=\mathbf{a}_{1}+2 \mathbf{a}_{2}+3 \mathbf{a}_{3} .
\end{aligned}
$$

Re-arranging in terms of the vectors:

$$
\begin{aligned}
& \mathbf{a}_{0}=\mathbf{A} \\
& \mathbf{a}_{1}=\mathbf{T}_{A} \\
& \mathbf{a}_{2}=3[\mathbf{B}-\mathbf{A}]-2 \mathbf{T}_{A}-\mathbf{T}_{A} \\
& \mathbf{a}_{3}=2[\mathbf{A}-\mathbf{B}]+\mathbf{T}_{A}+\mathbf{T}_{B}
\end{aligned}
$$

Substituting back into Equation (1) we obtain:

$$
\mathbf{r}(u)=\mathbf{A}\left(1-3 u^{2}+2 u^{3}\right)+\mathbf{B}\left(3 u^{2}-2 u^{3}\right)+\mathbf{T}_{A}\left(u-2 u^{2}+u^{3}\right)+\mathbf{T}_{B}\left(-u^{2}+u^{3}\right),
$$

or in matrix form:

$$
\mathbf{r}(u)=\left[\begin{array}{llll}
1 & u & u^{2} & u^{3}
\end{array}\right]\left[\begin{array}{cccc}
1 & 0 & 0 & 0 \\
0 & 0 & 1 & 0 \\
-3 & 3 & -2 & -1 \\
2 & -2 & 1 & 1
\end{array}\right]\left[\begin{array}{c}
\mathbf{A} \\
\mathbf{B} \\
\mathbf{T}_{A} \\
\mathbf{T}_{B}
\end{array}\right]
$$

$\mathbf{r}(u)$ is, essentially, a Hermitian interpolant and the bracketed factors in equation (4) can be viewed as its basis functions - Figure 2 conveys an intuitive understanding of their effect on the shape of the interpolant. We now proceed to discuss the suggested airfoil parameterization scheme based on the formulation outlined above.

3

Submitted to the American Institute of Aeronautics and Astronautics 


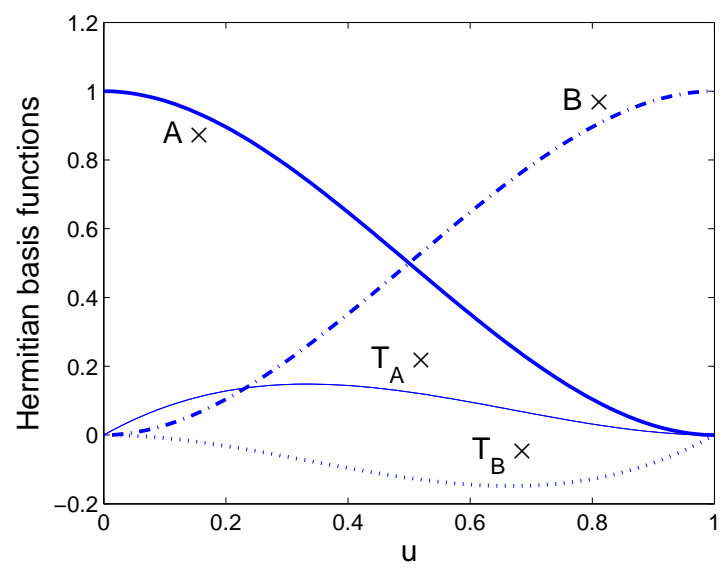

Fig. 2 The four basis functions of equation (4) shown alongside their respective multipliers.

\section{Airfoil Parameterization}

Describing and controlling airfoils via splines is, of course, not new; they are also used to smooth sets of coordinates ${ }^{8}$ resulting from other formulations or measurements. However, most such schemes involve the definition of a series of points or knots (see, for example, the NURBS design study reported in Ref. 9), which the spline interpolates or is defined by. This is a relatively awkward approach for two reasons.

First, setting up the ranges of the various design variables (node coordinates) is a far from trivial task, due to the interactions between the variables. For example, care must be taken for the bottom surface not to intersect the top surface, but, if thin, but reasonably cambered airfoils are to be included, this is made very difficult by the 'snaking' that often blights knot-based polynomial descriptions.

The second issue is that of parsimony. Each knot (or interpolation point, depending on the exact type of spline) has at least one degree of freedom, but two are often used. It is easy to see that this will not lead to a parameterization that is sufficiently parsimonious for conceptual design (for example, Lépine et al. ${ }^{9}$ settle on 11 variables - this appears to be one of the fewest encountered in the literature). It is worth remembering here the phenomenon sometimes referred to as the 'curse of dimensionality': if a certain level of design landscape information is acquired in a one-variable space by sampling it in $k$ locations, to achieve the same sample density in an $n$-dimensional space, $k^{n}$ observations are required.

Here we argue that the number of design variables can be reduced to six (assuming a sharp trailing edge, more on this later) while still maintaining a sufficiently broad coverage of the design space. We achieve this by not using any knots, other than the endpoints. The geometry is illustrated in Figure 3. The tangent of the upper surface $\mathbf{r}^{u}(u)$ in $\mathbf{A}$ (at the leading edge) is denoted by $\mathbf{T}_{A}^{\text {upper }}$ and its tangent in $\mathbf{B}$ by $\mathbf{T}_{B}^{\text {upper }}$ - the same logic is used for the nomenclature of the lower surface.

The shape of the airfoil is thus defined by the orientation and the magnitude of the tangent vectors. $\mathbf{T}_{A}^{\text {upper }}$ and $\mathbf{T}_{A}^{\text {lower }}$ will always be pointing vertically downwards and upwards respectively, with their magnitude defining the tension in the spline, thus controlling the 'bluntness' of the leading edge. $\alpha_{c}$, 


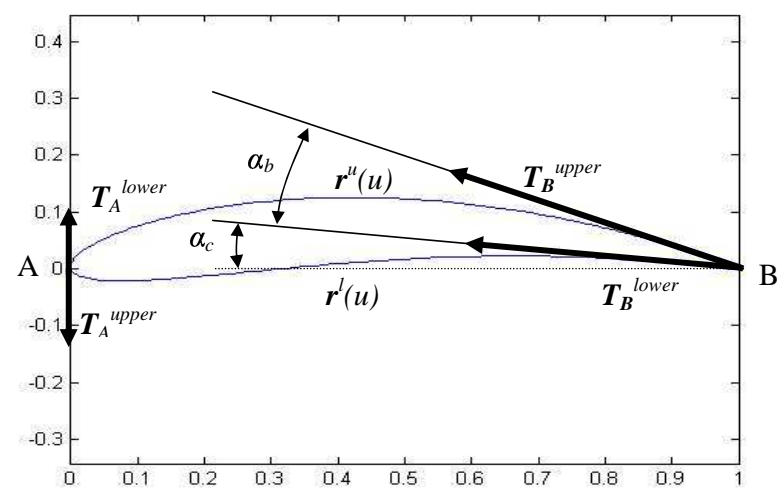

Fig. 3 The airfoil parameterization scheme based on two Ferguson splines. The parametric curves $\mathbf{r}^{u}(u)$ and $\mathbf{r}^{l}(u)$ describe the upper and lower surfaces respectively.

which might be called the camber angle, defines the orientation of $\mathbf{T}_{B}^{\text {lower }}$, while the boattail angle $\alpha_{b}$ determines the orientation of $\mathbf{T}_{B}^{\text {upper }}$. The magnitudes (tangent tensions) of these vectors determine the shape of the middle section of the airfoil.

It can be seen that, in addition to having relatively few parameters, they also relate intuitively to geometrical features of the airfoil and, to some extent, even to its aerodynamic features: the tensions in the tangents at $\mathbf{A}$ determine the angle of attack capability, the tangents at $\mathbf{B}$ are related to the pressure drag and drag rise characteristics and $\alpha_{c}$ has a major influence on $c_{l}$. Structurally, the boattail angle $\alpha_{b}$ will, together with the nose tangent magnitudes, control the cross-sectional area of the wing.

We conclude our discussion of the fundamentals of this airfoil definition scheme with a note on finite thickness trailing edges. Should such an airfoil be required, the curves $\mathbf{r}^{u}(u)$ and $\mathbf{r}^{l}(u)$ (see Fig. 3) will not meet in $\mathbf{B}$, but will have separate endpoints on a perpendicular raised to the chord in $\mathbf{B}$, at ordinates $\epsilon_{u}$ and $\epsilon_{l}$ respectively. This requires no change to the basic formulation, other than replacing $\mathbf{B}(1,0)$ with $\mathbf{B}^{u}\left(1, \epsilon_{u}\right)$ and $\mathbf{B}^{l}\left(1, \epsilon_{l}\right)$ in the definitions of the two surfaces of the airfoil.

These could be fixed at certain values, but can also be allowed to function as additional design variables. If this is deemed necessary in an optimization context, a seventh design variable can be added, say, $\epsilon$, where the the top and bottom surfaces are anchored at the trailing edge by $\mathbf{B}^{u}(1, \epsilon / 2)$ and $\mathbf{B}^{l}(1,-\epsilon / 2)$ respectively.

Another finite trailing edge scenario could be when we are seeking a pair of Ferguson splines that best fits an airfoil given by a set of points. The authors have found that, although this involves adding an eighth variable, the search process is facilitated by using separate $\epsilon_{u}$ and $\epsilon_{l}$ trailing edge definition variables. We make two final points before proceeding to demonstrate this type of exercise on a number of well-known airfoil sections. First, the search process can actually yield a sharp trailing edge approximation (i.e., $\epsilon_{u}=\epsilon_{l}$ ) to a finite trailing edge profile. A second, somewhat pedantic point is that if both $\epsilon_{l}$ and $\epsilon_{u}$ are positive or negative, $\mathbf{A B}$ will, technically, no longer be the chord of the 'clone' airfoil. 


\section{Reproducing an Existing Airfoil}

The fundamental test of any airfoil parameterization scheme is whether it can reproduce, or, more realistically, approximate to a reasonable accuracy, a fairly broad range of standard airfoil sections. We have generated a number of such Ferguson spline approximations by minimizing a difference measure between them and some well known target airfoils. This metric is purely geometrical: we used the sum of the squares of distances between the target and its Ferguson approximation over a fine mesh.

We have also compared the originals and their spline-based clones in terms of pressure distributions over them in flow conditions that, though to some extent arbitrary, were chosen to be roughly representative of real world applications of the airfoils in question. We used the Viscous Garabedian and Korn (VGK) ${ }^{10}$ two-dimensional full potential solver to compute the pressure profiles (more on VGK in Section V.).

It is perhaps worth pausing here to make two points before we proceed to present the results. We show pressure distributions (as well as the actual airfoil geometries) for both the original airfoil and its clone because, ultimately, that is an indication of how well the latter emulates the former in terms of its aerodynamic performance. This will, of course, be just that - and indication, as a "full" comparison would involve an infinity of combinations of Reynolds numbers, Mach numbers and angles of attack.

The second point is a somewhat philosophical one. One could clone an airfoil by minimizing the differences between the pressure distributions, rather than the geometries themselves, in a fashion reminiscent of inverse design. This would, however, be an unreasonably ample exercise for the reason mentioned earlier, as well as, in these authors' opinion, a less germane one from the perspective of what we are actually testing here: the flexibility of the airfoil parameterization scheme being proposed.

Considering now the actual comparisons, these indicate excellent agreement for a number of airfoils - in particular those used in fully subsonic applications - and reasonable agreement (though what can be considered "reasonable" is not obviously quantifiable) on aft-loaded airfoils designed for supercritical applications. Table 1 contains the data necessary for the reader to be able to reconstruct the airfoils being considered, while Figures 4 through 8 depict the geometrical and aerodynamic comparisons.

\begin{tabular}{|c|c|c|c|c|c|c|c|c|}
\hline Airfoil & $\left|\mathbf{T}_{A}^{\text {upper }}\right|$ & $\left|\mathbf{T}_{A}^{\text {lower }}\right|$ & $\left|\mathbf{T}_{B}^{\text {upper }}\right|$ & $\left|\mathbf{T}_{B}^{\text {lower }}\right|$ & $\alpha_{c}[\mathrm{deg}]$ & $\alpha_{b}[\mathrm{deg}]$ & $\epsilon_{l}$ & $\epsilon_{u}$ \\
\hline NACA 5410 & 0.1584 & 0.1565 & 2.1241 & 1.8255 & 3.8270 & 11.6983 & -0.0032 & 0.0012 \\
\hline NACA 21012 & 0.1674 & 0.2402 & 2.2482 & 1.3236 & -8.7800 & 17.2397 & -0.0074 & -0.0080 \\
\hline RD canard & 0.0231 & 0.0209 & 2.7965 & 3.0411 & -4.1414 & 19.5966 & -0.0026 & -0.0026 \\
\hline RAE 2822 & 0.1902 & 0.0000 & 1.1330 & 3.0504 & -6.3167 & 22.3472 & -0.0040 & -0.0040 \\
\hline SC(2)-0714 & 0.3529 & 0.4707 & 0.4543 & 0.2817 & 13.9566 & 16.8290 & -0.0096 & -0.0096 \\
\hline
\end{tabular}

Table 1 Standard airfoils and the design variables that generate the spline-based airfoil description of the best clones found by the authors. 
NACA 5410 airfoil, $\alpha=1 \mathrm{deg}$, Mach $=0.35, R e=1000000$
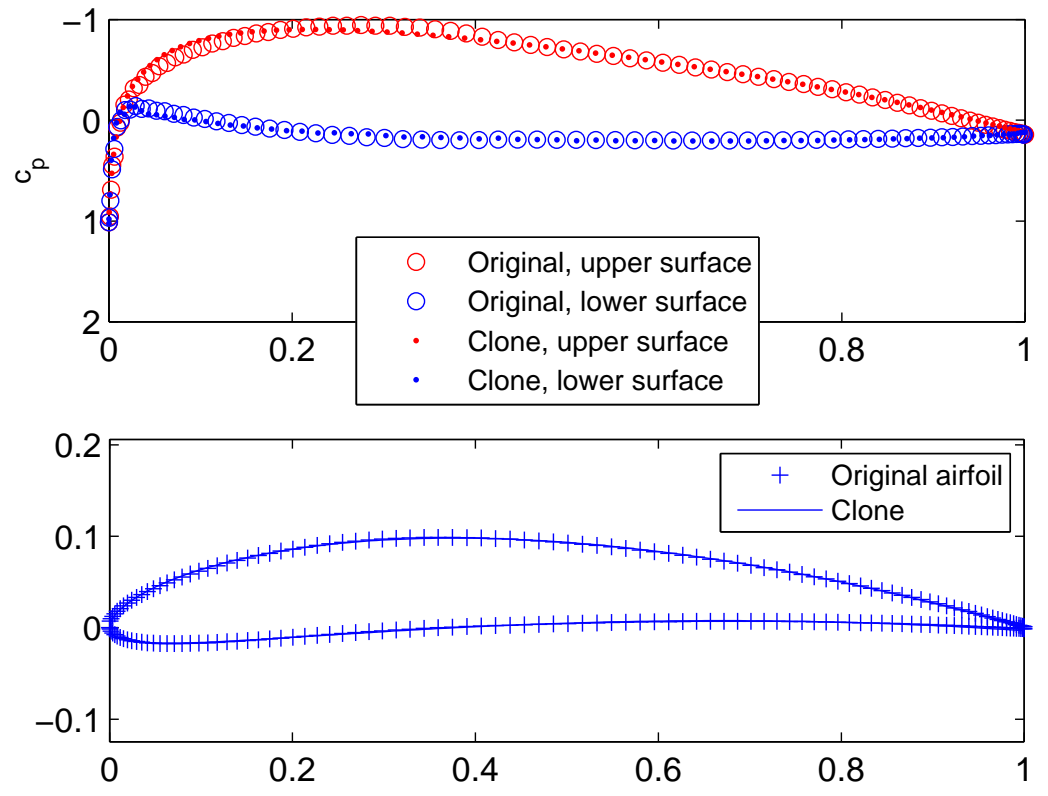

Fig. 4 Almost perfect agreement can be observed on the NACA four digit series.

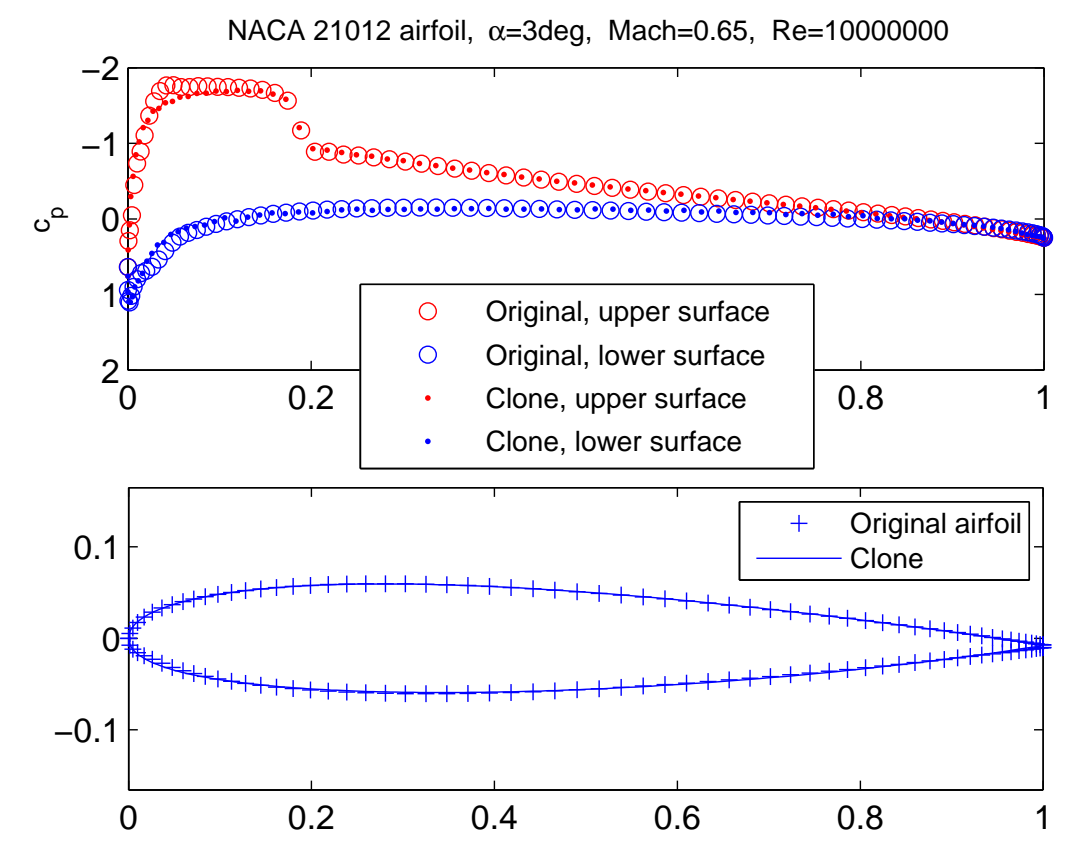

Fig. 5 Very good agreement on a five-digit NACA profile.

7

Submitted to the American Institute of Aeronautics and Astronautics 

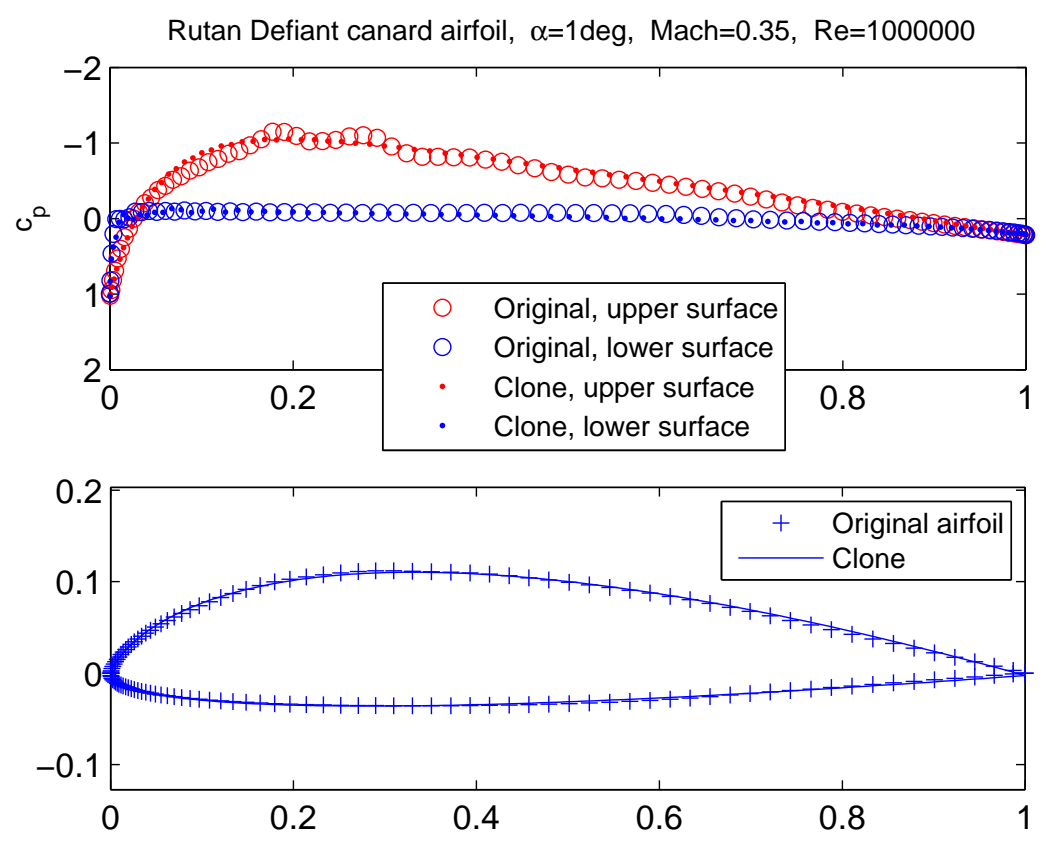

Fig. 6 Very good agreement on the airfoil of the Rutan Defiant canard.
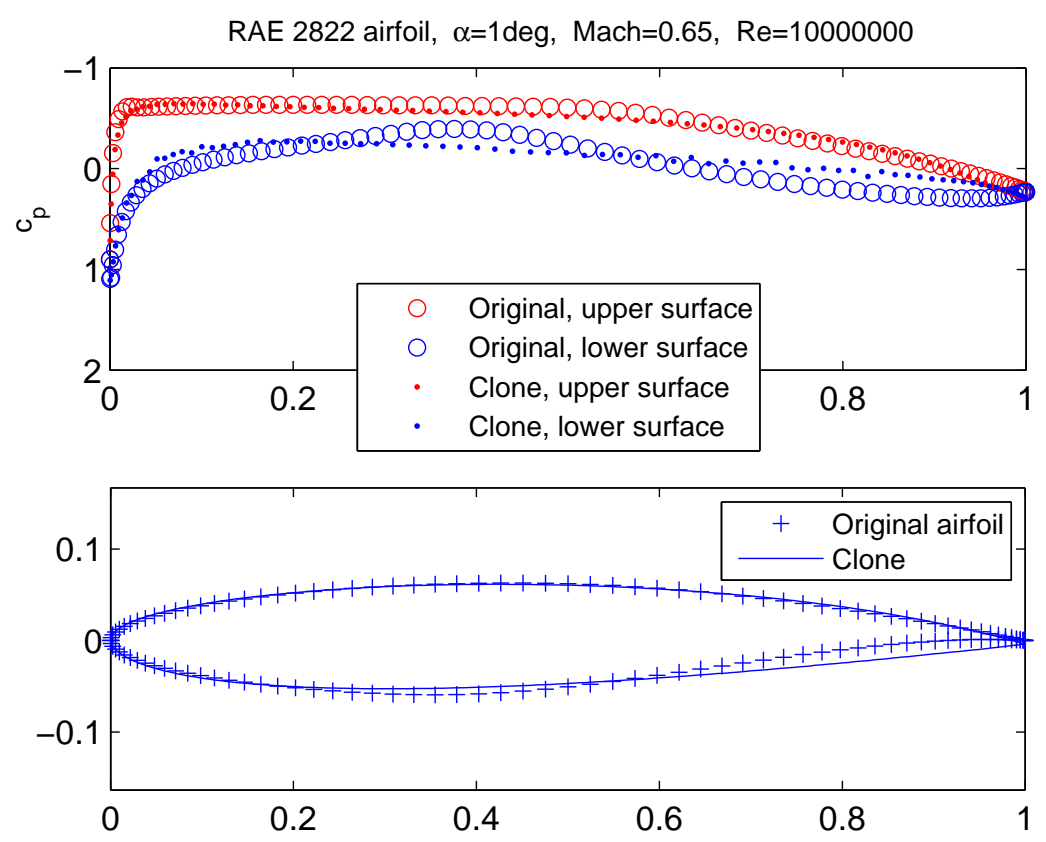

Fig. 7 Good agreement on the top surface of the RAE 2822 transonic airfoil, geometrical differences leading to slightly different lower surface pressure distributions.

8

Submitted to the American Institute of Aeronautics and Astronautics 
SC(2)-0714 airfoil, $\alpha=1$ deg, Mach=0.7, Re=10000000
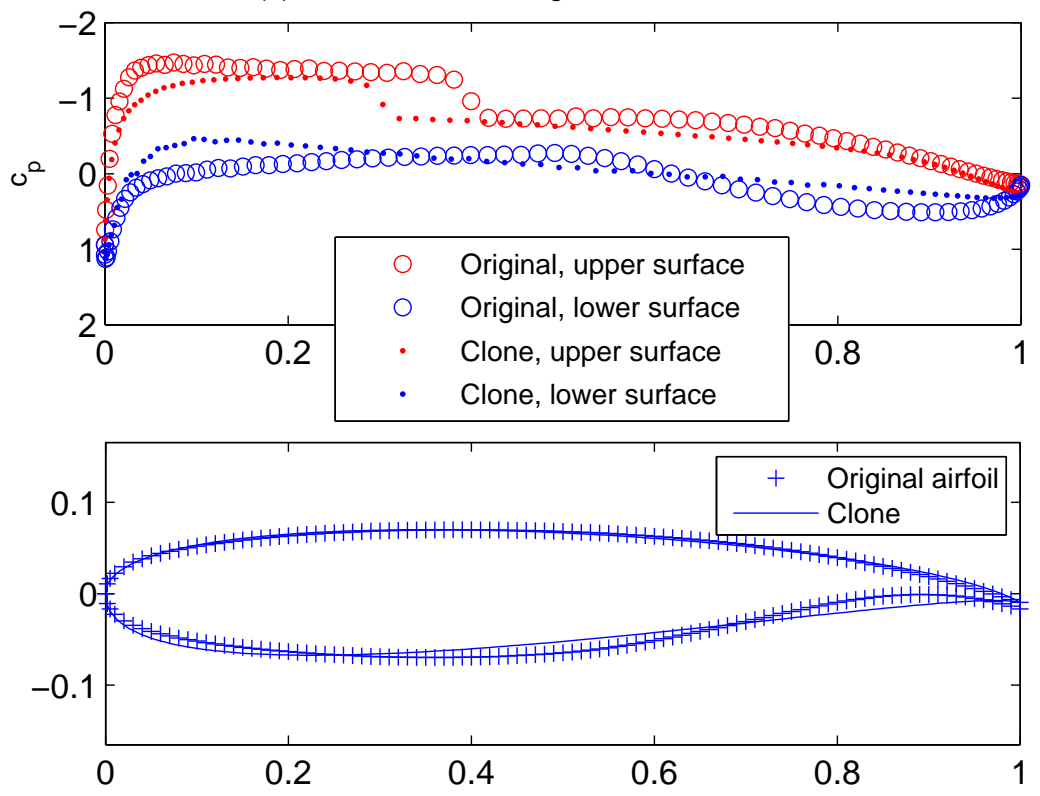

Fig. 8 The SC(2)-0714 supercritical airfoil: visible geometrical differences lead to deviations in the pressure profile of the clone.

\section{Notes on the Geography of the Design Space}

The fundamental trade-off in most design studies is between the size of the design space and the fidelity of the engineering analysis. Narrow ranges on the design variables allow the use of high computational cost analysis tools, but are unlikely to lead the designer to "unexpected", truly novel solutions - nor is this always required. For example, the preliminary or detail design phase of a project will seldom allow dramatic shape changes.

Conversely, conceptual studies may be guided by the output of light-weight computational analysis tools. Here a large design space is a must, as the analyst wishes to keep an open mind in terms of the shape of the aerodynamic surfaces under investigation. The ranges of the design variables have to reflect this.

In choosing lower and upper bounds for the six airfoil shape variables used in this paper we have opted for a somewhat arbitrary set of values that are more suited to the latter of the two possible scenarios outlined above. After all, once we get to know the broader picture, an understanding of the more conservative first case follows naturally. Table 2 contains these fairly broad ranges.

\begin{tabular}{|c|c|c|c|c|c|c|}
\hline Variable & $\left|\mathbf{T}_{A}^{\text {upper }}\right|$ & $\left|\mathbf{T}_{A}^{\text {lower }}\right|$ & $\left|\mathbf{T}_{B}^{\text {upper }}\right|$ & $\left|\mathbf{T}_{B}^{\text {lower }}\right|$ & $\alpha_{c}[\mathrm{deg}]$ & $\alpha_{b}[\mathrm{deg}]$ \\
\hline Lower bound & 0.1 & 0.1 & 0.1 & 0.1 & -15 & 1 \\
\hline Upper bound & 0.4 & 0.4 & 2 & 2 & 15 & 30 \\
\hline
\end{tabular}

Table 2 The ranges of the six airfoil definition variables, as used in this paper.

9

Submitted to the American Institute of Aeronautics and Astronautics 
So what are the defining features of the airfoil design space bounded by the values indicated in Table 2? First of all, $\alpha_{c}$ 's variation between -15 deg and 15 deg indicates the possibility of negatively cambered airfoils. The designer may wish to consider these for a variety of reasons, for example at certain stations along the wing of a flying wing aircraft (to ensure static stability). Approximately $26 \%$ of the design space considered here yields negative camber airfoils - the distribution of these, as a subset of a Sobol sequence* filling the design space, is illustrated in Figure 11 included in the Appendix.

It is also worth noting that around $1.7 \%$ of the design space defined by Table 2 leads to nonsensical designs - these are airfoils whose upper and lower surfaces intersect. Although the distribution of such a small number of unphysical designs throughout the design space is somewhat academic, we have included Figure 12 into the Appendix to illustrate that these failures cannot be eliminated altogether by simply tightening the bounds on the design variables.

\section{Drag Versus Structural Design Drivers - an Airfoil Design Study}

One of the fundamental multidisciplinary trade-offs of air vehicle design involve determining the maximum depth (or 'thickness') of an airfoil and its chordwise variation. The argument usually goes like this. A thick airfoil will present a larger apparent cross-section to the airflow and this will increase the pressure drag. Sometimes also termed form drag, this component of the overall drag will also be influenced by the chordwise variation of the thickness. The greater the thickness, however, the deeper a spar can be, thus yielding better structural efficiency, and, consequently, lower structural weight. Also, there is more room inside the wing, tail surface, canard, etc. defined by the airfoil - this space may be needed for fuel storage, hydraulic systems, wire looms, de-icing installations and so on. Conversely, thin airfoils, while often having low values of $c_{d}$ (at an angle of attack that will yield the required amount of lift) mean either heavy or light, but flutter-prone, flexible aerodynamic surfaces, with little space inside for vital systems.

We demonstrate the flexibility of the airfoil parameterization scheme proposed here, as well as the coverage of the design space bounded by the limits shown in Table 2, by conducting such a trade study. We construct a Pareto front involving two objectives. For a range of airfoils we calculate their maximum depth, that is, the maximum distance between their upper and lower surface. This is an important consideration in single main spar designs for the reasons mentioned earlier.

We also compute the drag $\left(c_{d}\right)$ for each airfoil, using VGK, a two-dimensional flow solver, which couples finite difference solutions of the inviscid flow about the airfoil with solutions for the displacement effects of the boundary layer and wake. The inviscid component is the solution of the full potential equations for steady, compressible flow. The viscous drag is estimated from the momentum thickness of the wake far downstream of the aerofoil. ${ }^{10}$

Coded in FORTRAN, a VGK solve takes around one second of computing time on a Pentium IV processor, including the conformal mapping-based mesh generation and the iterations required to establish the angle of attack needed to achieve a target lift value $-c_{l}=0.35$ in this case. This value, as

\footnotetext{
*A Sobol sequence is a pseudo-random, low discrepancy multi-dimensional set of numbers with the property that its first $n_{1}$ elements are distributed almost as uniformly as the first $n_{2}>n_{1}$, for reasonably large values of $n_{1}$. ${ }^{11}$
}

10

Submitted to the American Institute of Aeronautics and Astronautics 
well as the freestream flow set to Mach 0.2 and Re $7 \times 10^{6}$, our experiments are roughly representative of the 10,000ft cruise conditions of a light, unpressurized General Aviation aircraft.

To establish as accurate a picture of the maximum depth (which we wish to maximize) versus $c_{d}$ at $c_{l}=0.35$ (which we wish to minimize) trade-off front as possible, we have constructed a sampling plan of the design space defined in Table 2 consisting of the first 145,000 points of the Sobol sequence in six dimensions (only sharp trailing edge airfoils were considered). After eliminating designs that VGK failed to converge on, nonsensical (self-intersecting) designs and those airfoils which fell outside the 90 percentile range of $\left|c_{l}-0.35\right|$, we were left with a set of just over 130,000 points - these are represented on the scatter plot shown in Figure 9. The colour of each dot corresponds to the maximum absolute camber of the design in terms of percentage of maximum thickness. As we are considering a relatively low $c_{l}$, cruise condition, it is not surprising that low camber airfoils dominate the neighbourhood of the Pareto front.

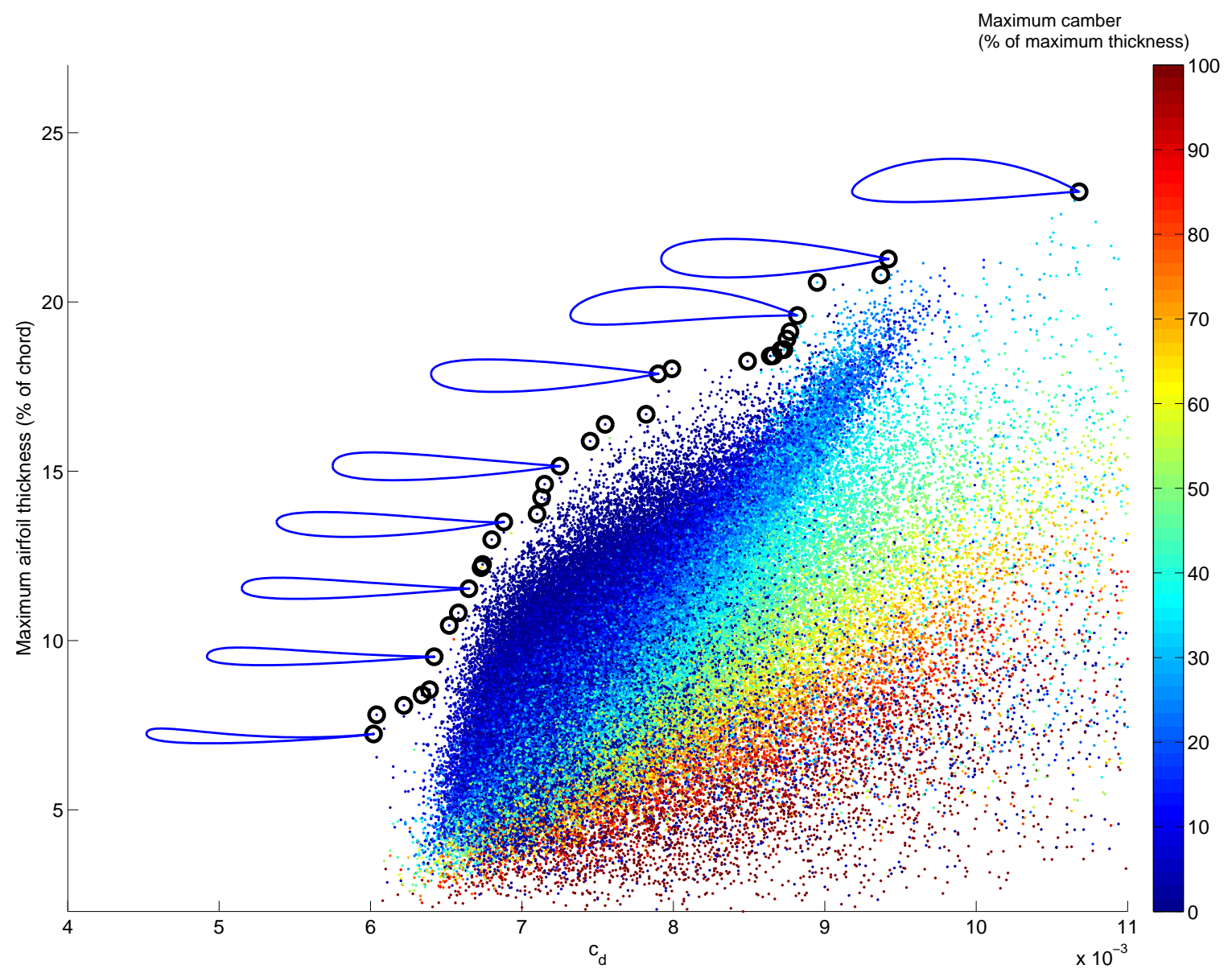

Fig. 9 Just over 130,000 airfoil designs generated according to a space-filling sampling plan. The black circles highlight non-dominated (Pareto-optimal) solutions of the airfoil thickness versus drag at $c_{l}=0.35$ trade-offs - some of these airfoils are are also depicted, positioned with their trailing edges on the corresponding point. The colors represent maximum camber.

11

Submitted to the American Institute of Aeronautics and Astronautics 


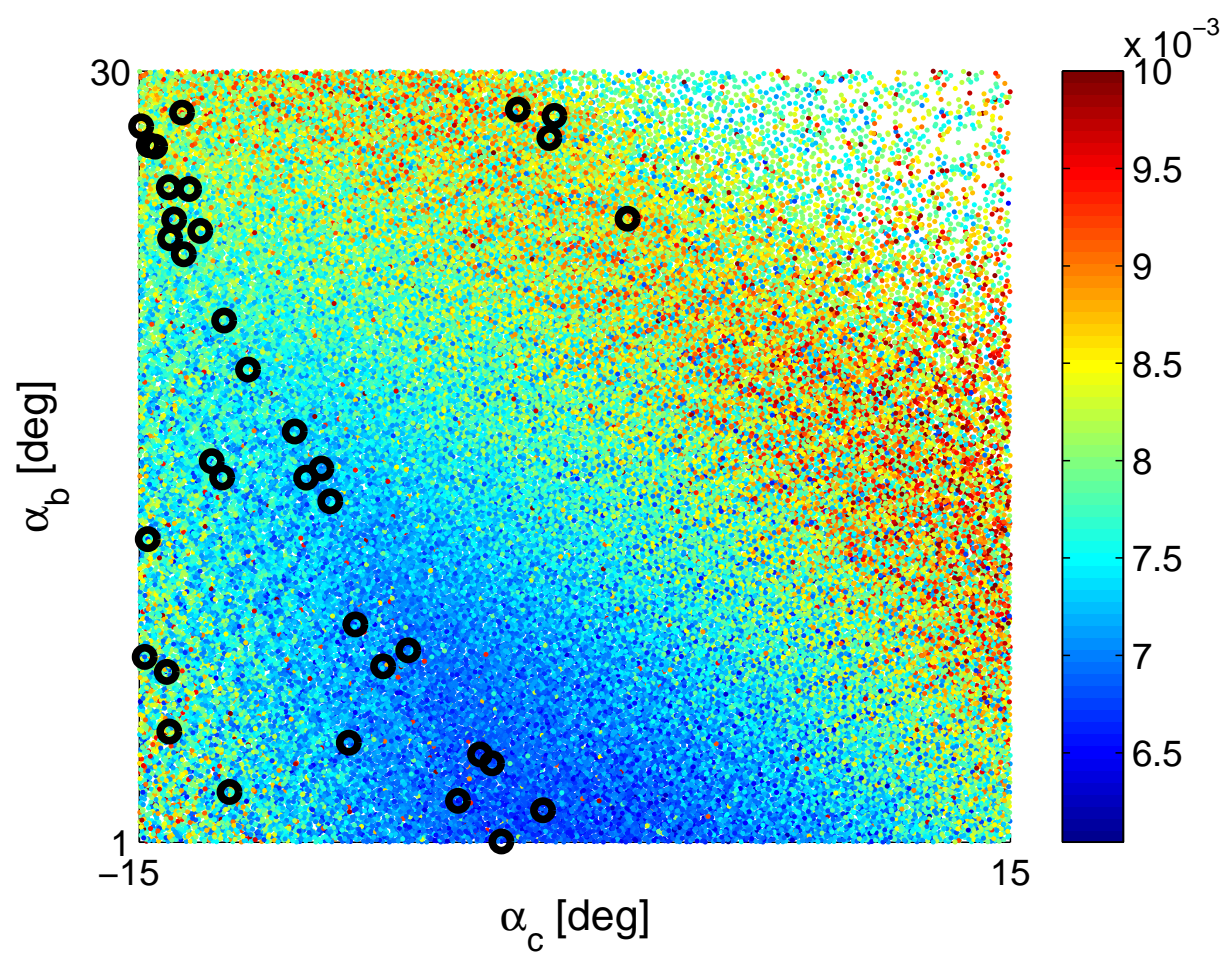

Fig. 10 A boattail angle versus camber angle section through the same dataset as that shown in Figure 9, the colors representing $c_{d}$. The black circles, as before, indicate the points of the maximum thickness versus $c_{d}$ Pareto front.

Figure 10 represents the same $c_{d}$ data, this time projected, along with the non-dominated points, onto a section of the design variable space: boattail angle $\alpha_{b}$ versus camber angle $\alpha_{c}$. We have already alluded to the fact that a good shape formulation will have a clear relationship between geometrical and aerodynamic features. Figure 10 makes this point from the perspective of the design variables $\alpha_{b}$ and $\alpha_{c}$.

Clearly, the combination of a sharp aft end (small boattail angle) and small camber ( $\alpha_{c}$ in the neighborhood of zero) yield the smallest drag coefficients. Red dots dominate the area above the diagonal, indicating that thick (in the large boattail angle sense) and highly cambered (large $\alpha_{c}$ ) airfoils will have high drag. However, their large thickness will have earned them places on the top end of the Pareto-front, as shown by the cluster of black circles in that area. The rest of the Paretopoints can be seen to fall below a line through the $\alpha_{b}$ vs. $\alpha_{c}$ space.

This is, of course, just one of the myriad of trade studies that that would, in a real-life design situation, yield the airfoil eventually used for this application. The present study ignores three-dimensional effects, stall characteristics, pitching moment and trim drag analyses, stability considerations, the requirement for a maximum angle of attack needed to achieve approach speed (to maintain runway visibility in case of the flaps failing to deploy), ground effect behavior, manufacturability, cost, etc. Its sole purpose is to illustrate the use of the spline parameterization scheme in a simple trade study. 


\section{Conclusions}

In the introduction we have promised to return to the 'wish-list' set out by Kulfan and Busoletti ${ }^{7}$ regarding airfoil parameterization schemes - we shall now reflect on the degree to which the scheme discussed here satisfies those criteria.

First, cubic polynomials are smooth and, as the scheme employed here does not use knots other than the endpoints, always well-behaved - that is, they do not show snaking-type instability (1). Due to its simplicity, the shape generation process is mathematically efficient (2). We have shown that as few as six design variables allow realistic trade-off studies and with eight variables a range of well-known airfoils can be cloned. We make the disclaimer here though, that the scheme is not flexible enough for a very accurate cloning of supercritical sections - this should, however, not be an impediment in its use as a conceptual level parameterization scheme (3). Further, as discussed in Section III, key design parameters such as leading edge shape, boattail angle and closure can be specified directly (4) - this allows easy and intuitive control and editing $(5,6)$. All of the types of geometries discussed here were considered under the umbrella of the same formulation - hence the consistency criterion met (7). Finally, the parametric definition of the Hermitian bases permits easy geometrical transformations, regardless of the chosen Cartesian frame of reference (8).

Thus, using a pair of simple Ferguson splines to represent airfoils, while not a universally applicable scheme (less well suited to detail design, particularly of transonic applications), is a parsimonious way of describing airfoils, which comes with the bonus of being natural and easy to implement in a CADbased design system.

\section{Acknowledgements}

The authors would like to thank BAE Systems and the Engineering and Physical Sciences Research Council (EPSRC) for the financial support of this work. We also gratefully acknowledge the help and much appreciated insights of Alexander Forrester and Tom Barrett.

\section{References}

${ }^{1}$ Wegman, E. J. and Wright, I. W., "Splines in Statistics," Journal of the American Statistical Association, Vol. 78, No. 382, 1983, pp. 351-365.

${ }^{2}$ Kochanek, D. H. U. and Bartels, R. H., "Interpolating Splines with Local Tension, Continuity and Bias Control," Computer Graphics, Vol. 18, No. 3, 1984, pp. 33-41.

${ }^{3}$ Ferguson, J., "Multivariable Curve Interpolation," Journal of the Association for Computing Machinery, Vol. 11, No. 2, 1964, pp. 221-228.

${ }^{4}$ Hicks, R. M. and Henne, P. A., "Wing Design by Numerical Optimization," Journal of Aircraft, Vol. 15, 1978, pp. $407-412$.

${ }^{5}$ Robinson, G. M. and Keane, A. J., "Concise Orthogonal Representation of Supercritical Airfoils," Journal of Aircraft, Vol. 38, No. 3, 2001.

${ }^{6}$ Sobieczky, H., "Parametric Airfoils and Wings," Notes on Numerical Fluid Mechanics, Fuji, K. and Dulikravich, G. S. (Eds.), Vol. 68, 1998, pp. 71-88.

${ }^{7}$ Kulfan, B. M. and Bussoletti, J. E., "“Fundamental” Parametric Geometry Representations for Aircraft Component Shapes," AIAA 2006-6948, 2006.

${ }^{8} \mathrm{Li}$, H. and Krist, S., "Spline-Based Airfoil Curvature Smoothing and Its Applications," Journal of Aircraft, Vol. 42, No. 4, July-August 2005, pp. 1065-1074.

13

Submitted to the American Institute of Aeronautics and Astronautics 
${ }^{9}$ Lepine, J., Guibault, F., J-Y., T., and Pepin, F., "Optimized Nonuniform Rational B-Spline Geometrical Representation for Aerodynamic Design of Wings," AIAA Journal, Vol. 39, No. 11, 2001, pp. 2033-2041.

${ }^{10}$ Freestone, M. M., "Transonic Data Memorandum: VGK method for two-dimensional aerofoil sections," Data Item 96028, ESDU International, October 1996.

${ }^{11}$ Sobol, I. M., A Primer for the Monte Carlo Method, CRC Press, 1994.

\section{Appendix}

The following two figures illustrate aspects of the geography of the design space of sharp trailing edge airfoils determined by the variable ranges indicated in Table 2 .
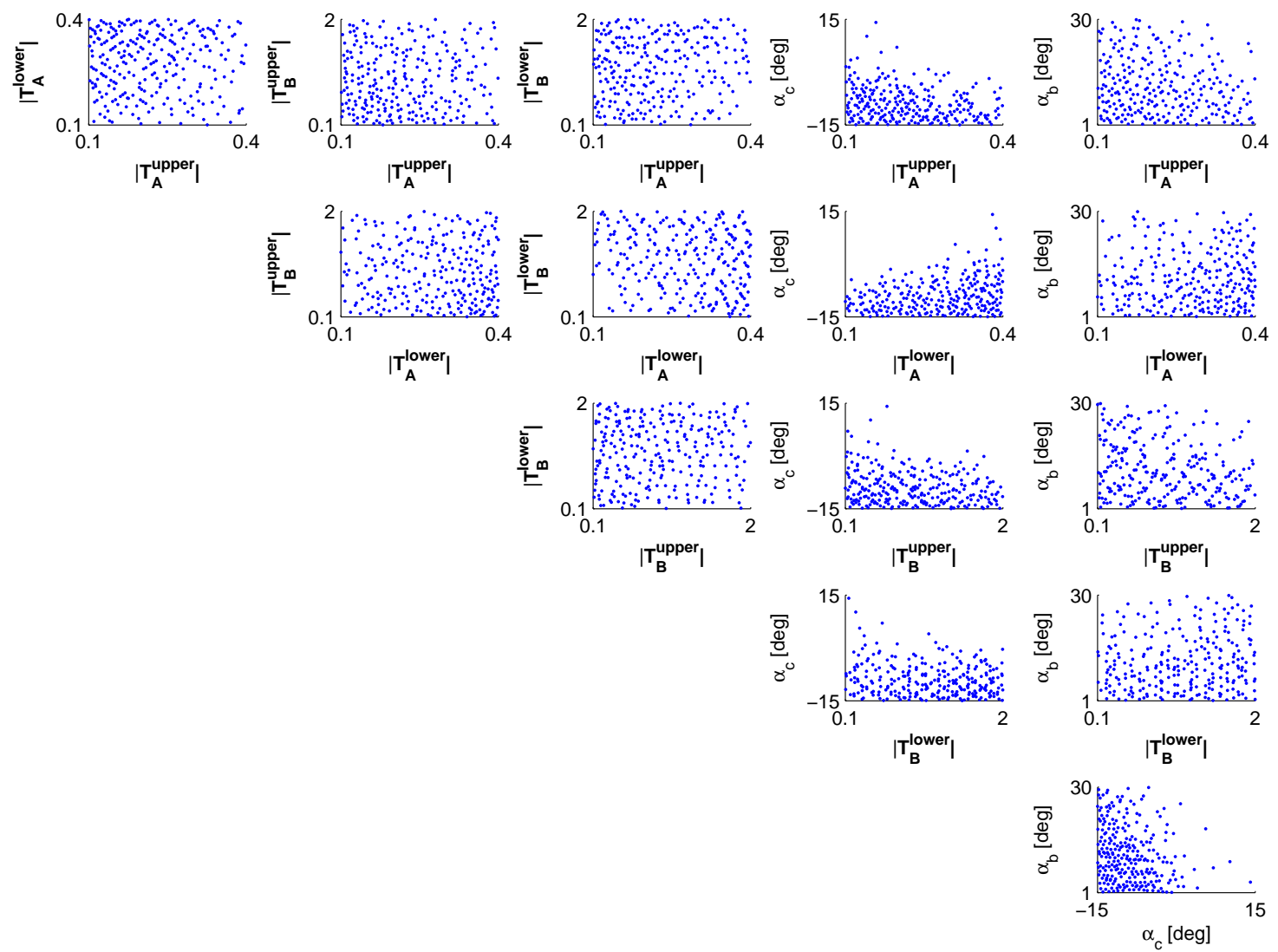

Fig. 11 Airfoils generated from 261 points of the first 1,000 points of a six-dimensional Sobol sequence have negative camber - the figure illustrates the distribution of these 261 points across the design space. 

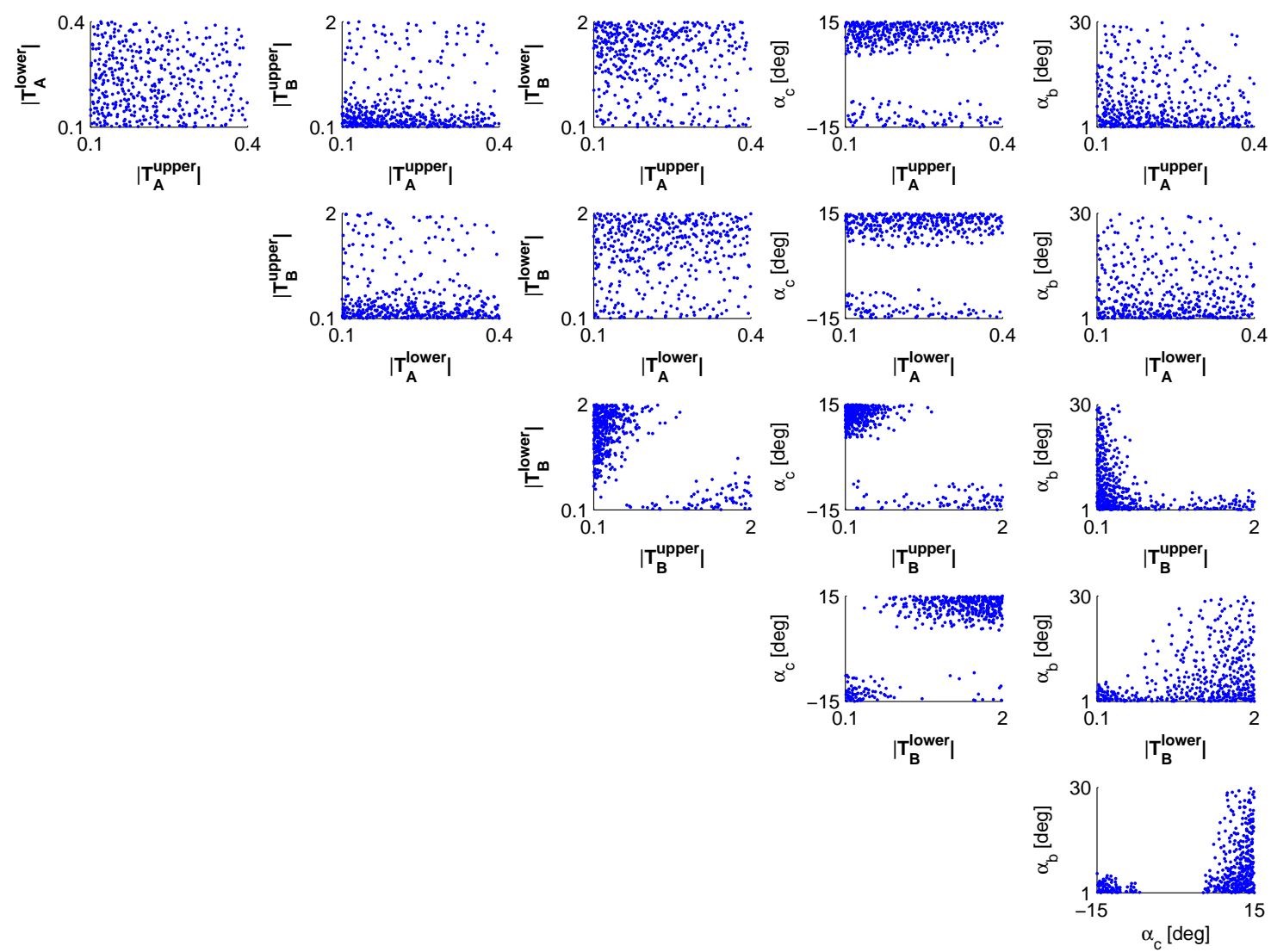

Fig. 12 Airfoils generated from 428 points of the first 25,000 points of a six-dimensional Sobol sequence are nonsensical on account of their lower and upper surfaces intersecting - the figure illustrates the distribution of these 428 points across the design space. 